\title{
Regulation of Ciliated Cell Differentiation in Cultures of Rat Tracheal Epithelial Cells
}

\author{
Alan B. Clark, Scott H. Randell, Paul Nettesheim, Thomas E. Gray, Bob Bagnell, \\ and Lawrence E. Ostrowski \\ Laboratory of Pulmonary Pathobiology, Airway Cell Biology Group, National Institute of Environmental Health \\ Sciences, Research Triangle Park, and Department of Pathology, University of North Carolina at Chapel Hill, \\ Chapel Hill, North Carolina
}

\begin{abstract}
The cellular pathway of ciliated cell differentiation and its regulation is poorly defined. To begin to understand the process of ciliated cell differentiation, we sought to identify factors regulating ciliated cell development in vitro. Rat tracheal epithelial (RTE) cells were cultured on collagen gel-coated membranes at an air-liquid interface in hormone- and growth factor-supplemented medium (complete medium [CM]). Under these conditions, RTE cells first proliferate and then differentiate into a pseudostratified mucociliary epithelium. Ciliated cell differentiation was measured using a monoclonal antibody, RTE3, which was shown to specifically react with the plasma membrane of ciliated cells. Cultures were immunostained in situ, and the percentage of the culture surface covered with ciliated cells was estimated using videomicroscopy and an image analysis program. If an air-liquid interface was not created and the cells were maintained in the submerged state, ciliated cell differentiation was suppressed 25 -fold. Culture in the absence of mitogenic components present in CM, including epidermal growth factor (EGF), cholera toxin (CT), or bovine pituitary extract, resulted in 2- to 4-fold increases in the percentage of ciliated cells. When both EGF and CT were removed from the media, DNA synthesis and total cell number was reduced, while ciliated cell differentiation increased as much as 5-fold. These results demonstrate that submersion inhibits, while withdrawal of mitogenic compounds promotes, ciliated cell differentiation in vitro.
\end{abstract}

Ciliated cells are one of the three main cell types lining the surface of the conducting airways. Individuals with primary ciliary dyskinesia have a genetic defect that results in abnormal axonemal structure and ciliary dysfunction. These individuals suffer from repeated pulmonary infections, demonstrating the importance of ciliated cells to the defense mechanisms of the lung (1). In addition to providing the motive force essential for mucociliary clearance, ciliated cells may produce antimicrobial peptides (2), function in immune surveillance (3), and regulate fluid and electrolyte transport (4).

As a result of their position at the surface of the airways, ciliated cells are exposed to many chemical and biological insults. Recent studies indicate that ciliated cells are easily

(Received in original form July 11, 1994 and in final form October 26, 1994) Address correspondence to: Dr. Lawrence E. Ostrowski, Pulmonary Path Lab., Mail Drop D2-01, N.I.E.H.S., P.O. Box 12233, Research Triangle Park, NC 27709.

Abbreviations: bovine pituitary extract, BPE; bovine serum albumin, BSA; complete media, CM; cholera toxin, CT; Dulbecco's modified Eagle's medium, DMEM; epidermal growth factor, EGF; hydrocortisone, HC; monoclonal antibody, mAb; phosphate-buffered saline, PBS; rat tracheal epithelial, RTE; scanning electron microscopy, SEM; trichloroacetic acid TCA; transmission electron microscopy, TEM.

Am. J. Respir. Cell Mol. Biol. Vol. 12. pp. 329-338, 1995 damaged or lost after exposure to air pollutants such as nitrogen dioxide, formaldehyde, and smoke (5-7). After damage to the tracheal epithelium, ciliated cells, which are believed to be terminally differentiated, must be regenerated to restore the normal mucociliary epithelium. While there is some evidence that secretory cells may serve as the precursor for ciliated cells after injury to the trachea (8), the regulation of ciliated cell differentiation has not been studied in detail.

One of the difficulties in studying ciliated cell differentiation has been the lack of a suitable cell culture model. With the improvements in tissue culture techniques over the past decade, including the use of exogenous extracellular matrix and the ability to culture cells on porous matrices, several groups have reported the presence of ciliated cells in cultures of airway epithelium (9-15). In some of these studies, it is unclear whether de novo ciliated cell differentiation occurs or whether pre-existing ciliated cells are simply maintained $(9,10)$. Our laboratory has recently described conditions that support mucociliary differentiation of rat tracheal epithelial (RTE) cells in vitro (16). RTE cells obtained by enzymatic digestion are seeded at low density on collagen gelcoated membranes in hormone- and growth factor-supplemented medium. Initially, the cells proliferate rapidly to form a monolayer of large, flat, undifferentiated cells. Proliferation then decreases and, after creation of an air-liquid 
interface, the cells undergo differentiation to produce a pseudostratified mucociliary epithelium containing mature secretory and ciliated cells. This process reproduces many of the features observed in vivo in models of tracheal wound healing or regeneration of tracheal grafts $(17,18)$.

In the present study, our aims were to develop a method to rapidly quantitate ciliated cell differentiation in cultures of RTE cells and to identify factors regulating ciliated cell differentiation in vitro. We demonstrate that the extent of ciliated cell differentiation can be measured using a monoclonal antibody (mAb), RTE3, which was shown to react specifically with the apical plasma membrane of ciliated cells by immunogold labeling. When cultures were maintained in the submerged state instead of at an air-liquid interface, ciliated cell differentiation was strongly suppressed. Removal of epidermal growth factor (EGF), cholera toxin (CT), or bovine pituitary extract (BPE) from the media resulted in 2- to 4-fold increases in the number of ciliated cells observed. DNA synthesis and total cell number were reduced in cultures grown in media lacking both EGF and $\mathrm{CT}$, while the percentage of surface area covered by ciliated cells increased. These studies have identified submersion and mitogenic compounds as important factors regulating ciliated cell differentiation.

\section{Materials and Methods \\ Materials}

Tissue culture media and reagents were from Sigma Chemical Co. (St. Louis, MO) except for EGF, transferrin, and rat tail collagen, which were purchased from Collaborative Research (Bedford, MA). Transwell-col tissue culture inserts (24-mm diameter, 0.4- $\mu \mathrm{m}$ pore size), referred to as "culture membranes," were obtained from Costar (Cambridge, MA). Bovine pituitaries were purchased from Pel Freeze (Rogers, AR). $\left[{ }^{3} \mathrm{H}\right]$ thymidine was from Amersham (Arlington, IL).

\section{Cell Culture}

RTE cells were cultured as previously described (16). Briefly, RTE cells were obtained from male Fisher 344 rats (10 to 14 wk old) by overnight digestion with pronase. Cells were cultured on collagen gel-coated membranes in complete medium (CM), which is composed of Dulbecco's modified Eagle's medium (DMEM)/Ham's F12 supplemented with L-leucine $(0.45 \mathrm{mM}$, final concentration), L-lysine $(0.50$ $\mathrm{mM})$, L-glutamine $(6.5 \mathrm{mM})$, L-methionine $(0.12 \mathrm{mM})$, $\mathrm{MgCl}_{2}(0.30 \mathrm{mM}), \mathrm{MgSO}_{4}(0.40 \mathrm{mM}), \mathrm{CaCl}_{2}(1.05 \mathrm{mM})$, phenol red $(8.6 \mathrm{mg} /$ liter $), \mathrm{NaHCO}_{3}(1.2 \mathrm{~g} /$ liter $)$, insulin (10 $\mu \mathrm{g} / \mathrm{ml})$, hydrocortisone (HC; $0.1 \mu \mathrm{g} / \mathrm{ml})$, CT $(0.1 \mu \mathrm{g} / \mathrm{ml})$, transferrin $(5 \mu \mathrm{g} / \mathrm{ml}$; Collaborative Research), EGF (25 $\mathrm{ng} / \mathrm{ml}$; Collaborative Research), phosphoethanolamine (50 $\mu \mathrm{M})$, ethanolamine $(80 \mu \mathrm{M})$, HEPES $(30 \mathrm{mM})$, bovine serum albumin (BSA; $0.5 \mathrm{mg} / \mathrm{ml}$ ), penicillin-streptomycin (50 $\mathrm{U} / \mathrm{ml}-50 \mu \mathrm{g} / \mathrm{ml}$; GIBCO, Grand Island, NY) and BPE (1\%). The cells were cultured submerged in media for the first 7 days, at which time an air-liquid interface was created. Modified media was prepared by omitting the indicated components from CM. Other modifications to the published procedures are as described below. The collagen gel was formed on top of the culture insert by spreading $0.4 \mathrm{ml}$ of $0.3 \%$ type I collagen (rat tail collagen; Collaborative Re- search) over the surface of each membrane. A gauze pad saturated with $30 \%$ ammonium hydroxide was placed in the bottom of a $160-\mathrm{mm}$ petri dish and covered. The membranes were placed on the lid of a $100-\mathrm{mm}$ petri dish, which was then placed on top of the gauze pad and covered. After 3 min, the membranes were placed in the culture wells containing $3 \mathrm{ml}$ of sterile water. After 4 to $8 \mathrm{~h}$, the water was replaced with sterile DMEM/F12 and incubated overnight at room temperature. Before plating the RTE cells, the DMEM/F12 in the lower chamber was replaced with CM $(2.5 \mathrm{ml})$ containing $10 \%$ fetal bovine serum and $3 \mathrm{mg} / \mathrm{ml}$ BSA. The cells were plated at a density of $2.4 \times 10^{4}$ cells/ $\mathrm{cm}^{2}$. The apical (upper) and basal (lower) compartments contained 0.5 and $2.5 \mathrm{ml}$ media, respectively.

\section{Cell Counts}

For counting, cells were released from the collagen gelcoated membranes by incubation in a solution of $0.2 \%$ pronase E (type XIV; Sigma), 0.15\% trypsin-1.6 mM EDTA (GIBCO) at $37^{\circ} \mathrm{C}$ for $30 \mathrm{~min}$. The solution was added to the top and bottom compartments ( 0.5 and $2.0 \mathrm{ml}$, respectively). After removing the cell suspension $(0.5 \mathrm{ml})$, membranes were washed with $0.5 \mathrm{ml}$ of phosphate-buffered saline (PBS), which was pooled with the cell suspension. Cell clumps were disrupted by 10 passages through a 20-gauge needle. Cells were then counted in a hemocytometer.

\section{DNA Synthesis}

DNA synthesis was assayed as the amount of $\left[{ }^{3} \mathrm{H}\right]$ thymidine that was incorporated into trichloroacetic acid (TCA)-precipitable counts during a $24-\mathrm{h}$ period. The day before assaying, media was replenished with media containing $5 \mu \mathrm{Ci} / \mathrm{ml}$ of [methyl $-^{3} \mathrm{H}$ ] thymidine. After $24 \mathrm{~h}$, the labeled media was removed and the cultures were washed twice in PBS. The cells were released from the membranes by incubation in the $0.2 \%$ pronase $\mathrm{E}, 0.15 \%$ trypsin- $1.6 \mathrm{mM}$ EDTA solution. A small aliquot $(30 \mu \mathrm{l})$ was used for cell counts, and the remainder was lysed in a solution of $0.3 \mathrm{~N} \mathrm{NaOH}$ and $1 \%$ sodium dodecyl sulfate at room temperature. An equal volume of $20 \%$ TCA was added, and the solution was mixed by vortexing. The DNA was precipitated in an ice bath for 15 min and collected by filtration through Whatman GF/C filters. The filters were rinsed twice with $5 \mathrm{ml}$ of $10 \%$ TCA, rinsed once with $5 \mathrm{ml}$ of ethanol, dried, and counted in Ecolume scintillation fluid (ICN; Irvine, CA).

\section{Antibody Staining of Whole Mounts}

Cultures were prepared for antibody staining by washing twice in PBS and fixing for at least $30 \mathrm{~min}$ in a solution of $4 \%$ formaldehyde/0.1 M potassium phosphate buffer $(\mathrm{pH}$ 7.4). Fixed cultures were washed 3 times in PBS. All of the following incubations were at room temperature. Fixed cultures were blocked for $30 \mathrm{~min}$ in PBS containing 1\% BSA $5 \%$ normal goat serum, and $1 \%$ gelatin. After blocking, cultures were incubated for $60 \mathrm{~min}$ in PBS containing $1 \%$ BSA and $0.1 \%$ gelatin plus a 1:250 dilution of mAb RTE3 hybridoma supernatant. An equal dilution of NSl myeloma cell conditioned media containing $50 \mu \mathrm{g} / \mathrm{ml}$ of normal mouse serum IgG was used in place of RTE3 hybridoma supernatant as a negative control. The cultures were washed 3 times in 
PBS containing $1 \%$ BSA and $0.1 \%$ gelatin and incubated in the same buffer plus a 1:250 dilution of peroxidase conjugated goat anti-mouse IgG (Jackson Immunoresearch Laboratories, West Grove, PA) for $60 \mathrm{~min}$. The cultures were then washed 3 times with PBS followed by a single wash in $50 \mathrm{mM}$ Tris- $\mathrm{HCl}(\mathrm{pH}$ 7.6). Peroxidase labeling was visualized by incubating for $10 \mathrm{~min}$ in $50 \mathrm{mM}$ Tris- $\mathrm{HCl}(\mathrm{pH} 7.6)$ containing $0.2 \mathrm{mg} / \mathrm{ml}$ diaminobenzidine and $0.006 \%$ hydrogen peroxide. After the color reaction, the cultures were washed abundantly with water, cut away from their support, and whole-mounted on glass slides.

\section{Quantitation of Ciliated Surface Area}

The amount of surface area of the whole-mounts covered by RTE3-stained cells was determined using a CCD video camera mounted to a light microscope and interfaced with a Macintosh II computer and a video monitor. For each stained whole-mount, 11 measurements at low power were taken at 2-mm intervals across the diameter. The area to be measured was viewed on the video monitor. For each measurement, the video image was captured on the computer monitor using the NIH Image software program, and the threshold density was set to highlight the stained cells. The threshold density was systematically set by increasing the threshold until areas with no staining (background) began to be highlighted. The highlighted image was made binary to convert the image into black and white pixels, and the erode command was used to remove background pixels. The percentage of positive pixels was calculated, and the average was taken to represent the percentage of surface area covered by ciliated cells.

\section{Transmission Electron Microscopy (TEM) Immunolabeling}

Inserts for TEM immunolabeling were washed twice in PBS and fixed in $4 \%$ formaldehyde/ $0.1 \mathrm{M}$ potassium phosphate buffer. Blocking, RTE3 antibody incubation, and subsequent washings were as above. The second antibody labeling was with a 1:50 dilution of $18 \mathrm{~nm}$ gold-conjugated goat antimouse IgG in PBS containing $1 \%$ BSA and $0.1 \%$ gelatin for $60 \mathrm{~min}$. After second antibody labeling, the membranes were washed with $1 \%$ BSA in PBS, fixed with $2 \%$ glutaraldehyde in $0.1 \mathrm{M}$ potassium phosphate buffer $(\mathrm{pH} 7.4)$, washed 3 times in $0.1 \mathrm{M}$ cacodylate ( $\mathrm{pH} 7.4$ ), incubated overnight in $1 \% \mathrm{OsO}_{4}$, dehydrated in ethanol and then in propylene oxide, and embedded in epon. Ultrathin sections were obtained and were counterstained with uranyl acetate and lead citrate.

\section{Cytospins}

Cells were released from the membrane following the procedure for cell counts. The cells were washed twice with PBS after the pronase-trypsin digestion and resuspended in $1.5 \mathrm{ml}$ of PBS per insert. After passing 10 times through a 20-gauge needle, $1.5 \mathrm{ml}$ of $4 \%$ formaldehyde $/ 0.1 \mathrm{M}$ potassium phosphate buffer was added to the suspension. Approximately $5 \times 10^{4}$ cells were centrifuged onto silanated slides (Digene, Diagnostics, Beltsville, MD). Slides were treated in $0.6 \%$ hydrogen peroxide in methanol for $10 \mathrm{~min}$ before immunostaining with RTE3. After immunostaining, the cytospin preparations were counterstained with $1 \%$ methyl green.
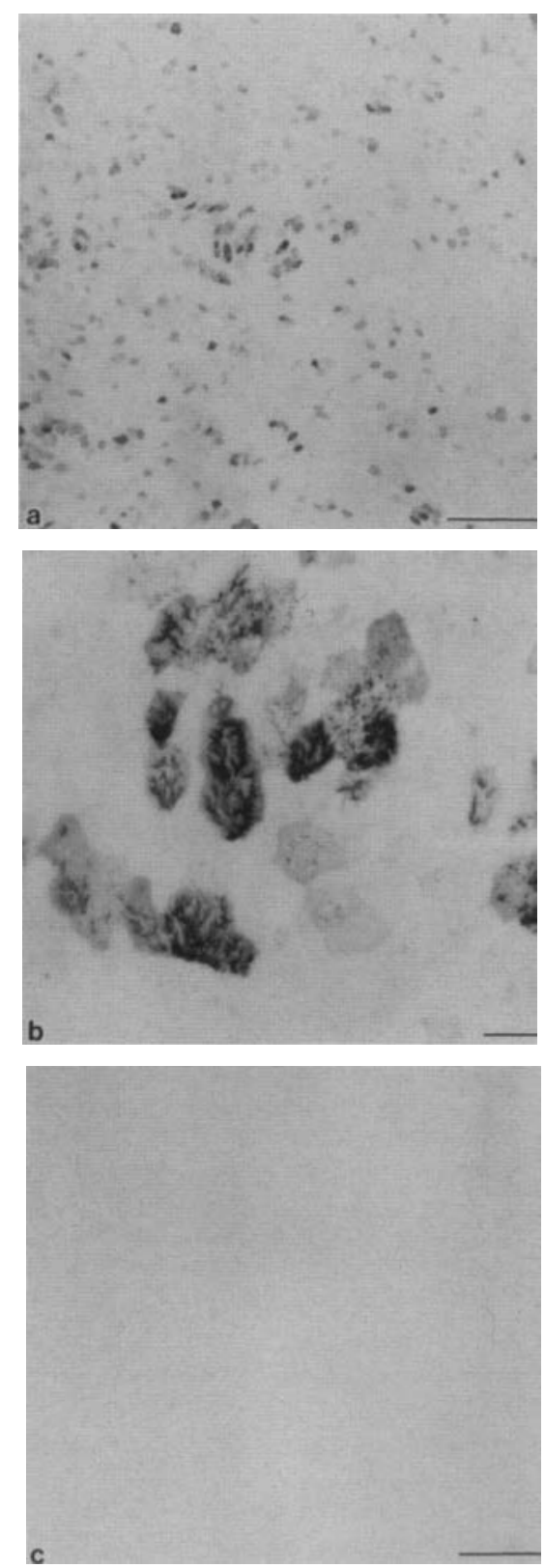

Figure 1. Immunostaining of air-liquid interface cultures with $\mathrm{mAb}$ RTE3. Interface cultures of RTE cells were fixed, immunostained with mAb RTE3, and whole-mounted. a. Low-power micrograph of a day 14 culture showing individual and groups of mAb RTE3positive cells. $b$. Higher magnification of an area in panel $a$, in which cilia are clearly visible on the surface of the stained cells. c. The negative control shows negligible background staining. Panels a and c, bars $=0.2 \mathrm{~mm}$; panel b: bar $=20 \mu \mathrm{m}$.

\section{Scanning Electron Microscopy (SEM)}

Cultures were fixed in situ with $3 \%$ glutaraldehyde, dehydrated in ethanol, and critical point-dried in liquid $\mathrm{CO}_{2}$. The culture membrane was detached from the plastic holder and mounted on an aluminum sample disk with silver paste. Samples were coated with $\mathrm{Au} / \mathrm{Pd}(60: 40)$ and analyzed in a Cambridge Stereoscan 200 (Leica, Inc., Deerfield, IL) at an accelerating voltage of $20 \mathrm{kV}$. 

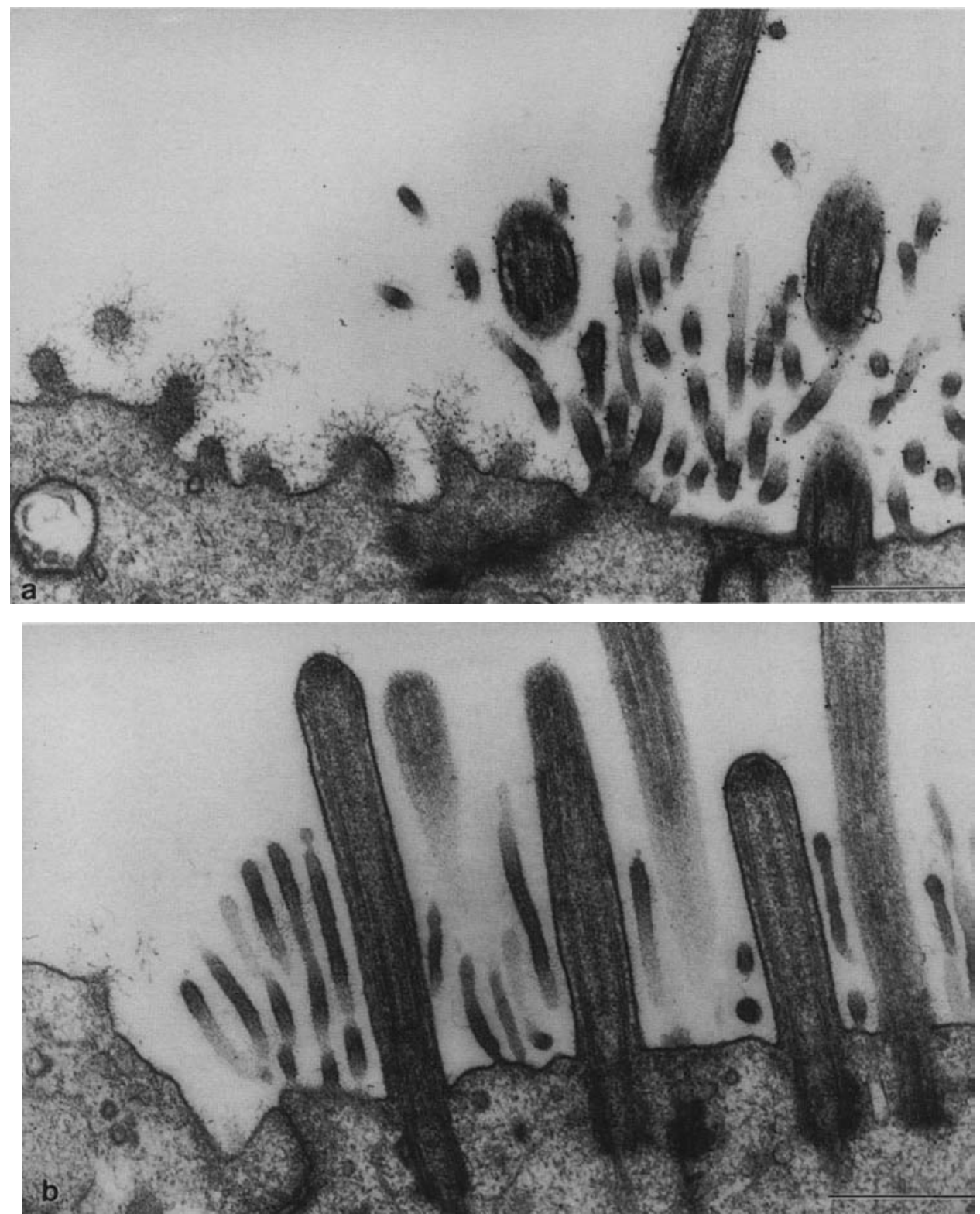

Figure 2. Ultrastructural localization of RTE3 antigen. Primary cultures of RTE cells were reacted with the mAb RTE3 (a) or control antibody (b), followed by incubation with gold-conjugated goat anti-mouse IgG, and then processed for TEM. a. A representative photomicrograph of a ciliated cell with mature cilia, microvilli, and basal bodies is shown next to a nonciliated cell. The ciliated cell shows clear labeling of the cilia, microvilli, and apical membrane with gold beads. The adjacent nonciliated cell is not labeled. b. Background staining with the negative control antibody was negligible. Bars $=0.5 \mu \mathrm{m}$.

\section{Quantitation of Mucin Production}

Mucin production was measured using a mAb, RTE11 (19), which reacts with rat mucin. Briefly, samples were collected by rinsing the apical surface of cells with PBS. Rat mucin standard was purified from hyaluronidase-digested apical secretions from day 14 to 21 RTE cultures by elution in the void volume from a Sepharose CL-4B column. For sandwich enzyme-linked immunosorbent assays, 96-well plates ( $\$ 3590$; Costar) were coated overnight at $4^{\circ} \mathrm{C}$ with $0.5 \mu \mathrm{g} /$ well $\mathrm{mAb}$ RTE11 in $0.9 \% \mathrm{NaCl}, 0.1 \mathrm{M} \mathrm{NaHCO}_{3}, \mathrm{pH} 9.5$, then rinsed twice with $20 \mathrm{mM}$ Tris, pH 7.6, $137 \mathrm{mM} \mathrm{NaCl}, 0.05 \%$ Tween-20 (TBST). Wells were blocked with $5 \%$ milk in TBST for $1 \mathrm{~h}$ at room temperature. Samples or standards were diluted in $1 \%$ milk-TBST and added to antibody-coated wells for $30 \mathrm{~min}$ at room temperature. Wells were washed with TBST and then a 1:500 dilution of peroxidase-conjugated rabbit anti-mucin in 1\% milk-TBST was added to the wells for a 30-min room temperature incubation. After washing again with TBST, color was developed with 0.4 $\mathrm{mg} / \mathrm{ml} O$-phenylenediamine- $\mathrm{HCl}, 0.012 \%$ hydrogen peroxide, $40 \mathrm{mM}$ citric acid, $0.1 \mathrm{M} \mathrm{Na}_{2} \mathrm{HPO}_{4}$, $\mathrm{pH}$ 5.0. The reaction was stopped with $\mathrm{HCl}$, and absorbance was measured at $490 \mathrm{~nm}$ in a plate reader.

\section{Results}

\section{mAb RTE3 Stains Ciliated Cells in RTE Cultures}

mAb RTE3 has been shown to react specifically with ciliated cells of the rat tracheobronchial epithelium (19). Interface cultures of RTE cells were immunostained in situ with $\mathrm{mAb}$ RTE3 and examined by light microscopy. At low power, single cells or clusters of cells reacting with RTE3 were apparent (Figure 1a). At high power, cilia were clearly visible on most stained cells (Figure 1b). Background staining with the negative control antibody was negligible (Figure lc). 


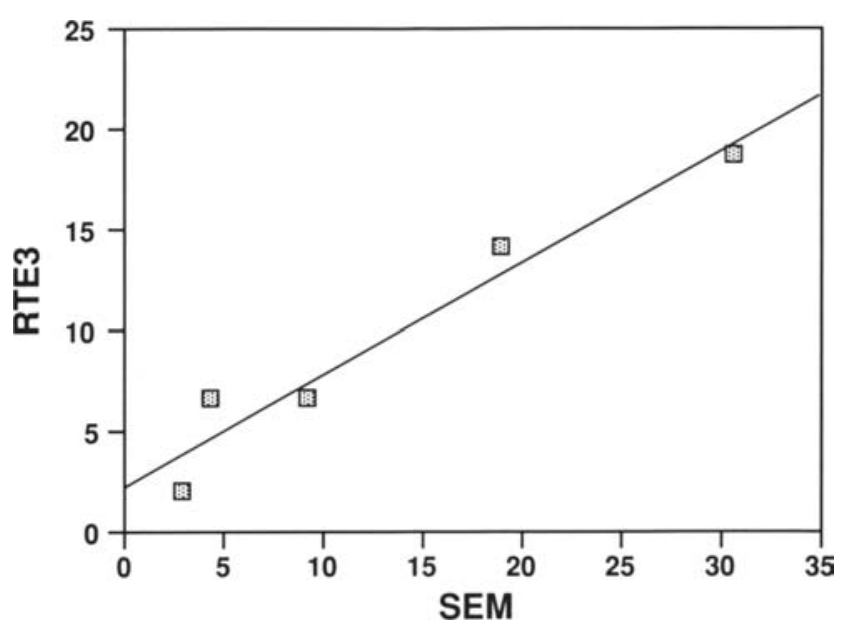

Figure 3. Correlation between RTE3 and SEM measurements of ciliated surface area. Cultures of RTE cells were immunostained with RTE3, and the percentage of surface area ciliated was estimated. The identical filters were processed for SEM, and the percentage of surface area ciliated was estimated from a series of photomicrographs at $1,000 \times$. Each point shows an individual culture membrane and represents the average of at least 10 individual measurements by SEM and RTE3. The line shown was derived by linear regression. The correlation coefficient between the two methods was 0.97 .

\section{mAb RTE3 Recognizes a Surface Antigen Expressed on Ciliated Cells}

To determine more precisely the distribution of the antigen recognized by mAb RTE3, cultures were incubated with $\mathrm{mAb}$ RTE3 followed by incubation with gold-conjugated goat anti-mouse IgG. Thin sections were then examined by TEM. Labeling on the surface of the cilia, microvilli, and plasma membrane of ciliated cells was observed (Figure 2a). Labeling of nonciliated cells was negligible (Figure 2a), as was labeling with the control antibody (Figure 2b). This demonstrates that RTE3 reacts specifically with an antigen expressed on the apical membrane of ciliated cells.

\section{RTE3 Immunostaining Can Be Used to Quantitate Ciliated Cell Differentiation}

Air-liquid interface cultures were fixed, immunostained with RTE3, and whole-mounted on slides. Quantitation of the stained area was performed by image analysis, as described in Materials AND METHODS. The use of the immunostaining method to estimate the extent of ciliated cell differentiation was supported by the following studies. First, those cultures that showed an increase in the number of ciliated cells visible by phase microscopy during the culture period also showed an increase in RTE3 staining. Second, cultures grown under four different conditions (see below) were stained with RTE3 and the percentage of surface area covered with ciliated cells was estimated using the image analysis program. The same culture membranes were then processed for SEM, and a series of photomicrographs were taken at $1,000 \times$. The percentage of surface area covered by ciliated cells was then measured in the photomicrographs and compared with the estimates obtained by the RTE3 immunostaining procedure. The results using the two proce-
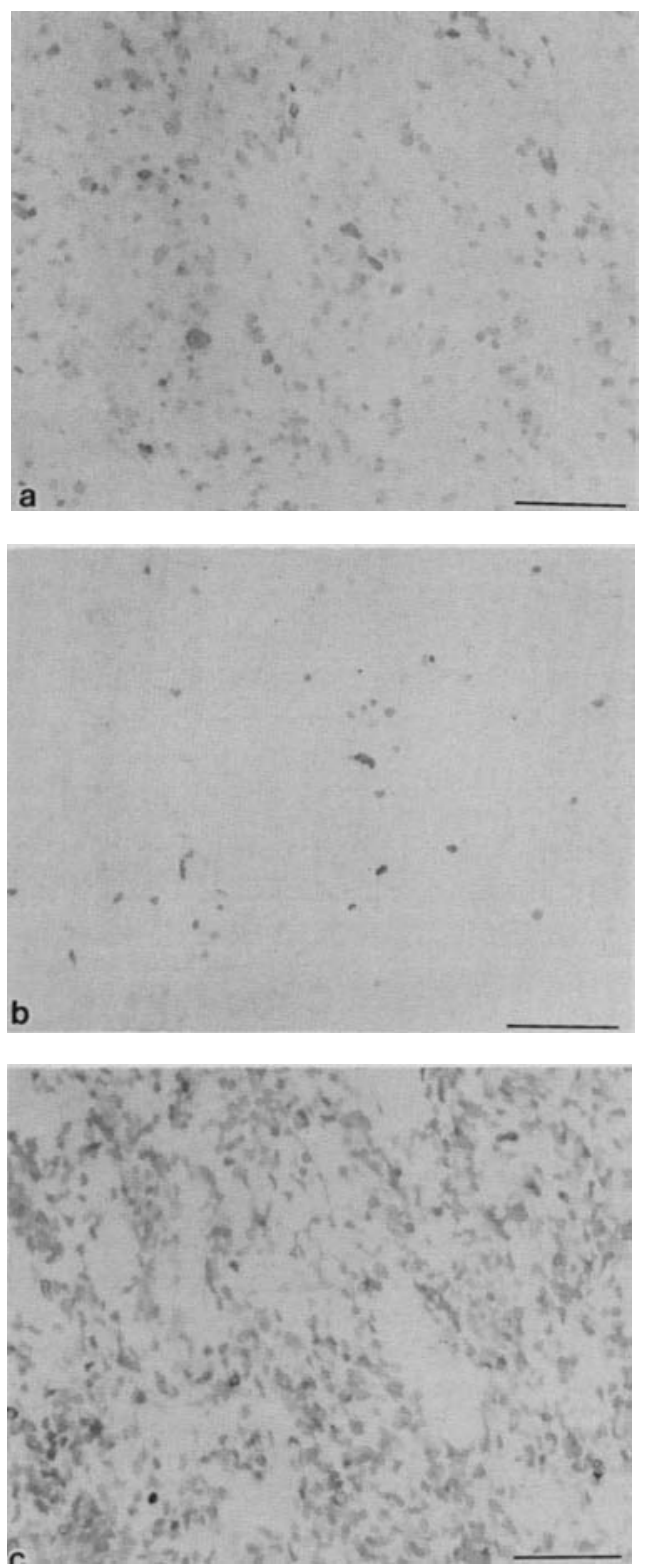

Figure 4. Immunostaining of RTE cell cultures grown under different conditions. RTE cells were cultured for 14 days under different conditions and were immunostained with mAb RTE3. Conditions shown include (a) air-liquid interface, CM, (b) submerged, CM, and (c) air-liquid interface, $\mathrm{CM}$ for days 1 through 7, and CM without EGF for days 7 through 14 . Bars $=0.2 \mathrm{~mm}$.

dures showed good agreement (Figure 3), with a correlation coefficient between the two methods of 0.97 . Finally, 200 RTE3-positive cells were randomly selected at low power. The culture membranes were scored using the image analysis system and then the individual cells were examined by high-power light microscopy $(1,000 \times)$. Some heterogeneity of staining was noted with the RTE3 antibody; some heavily ciliated cells appeared lightly stained, while some cells that possessed abundant microvilli but few or no cilia were heavily stained. Both lightly and heavily stained cells were included in the analysis. Greater than $90 \%$ of the cells scored positive by the RTE3 immunostaining procedure displayed 


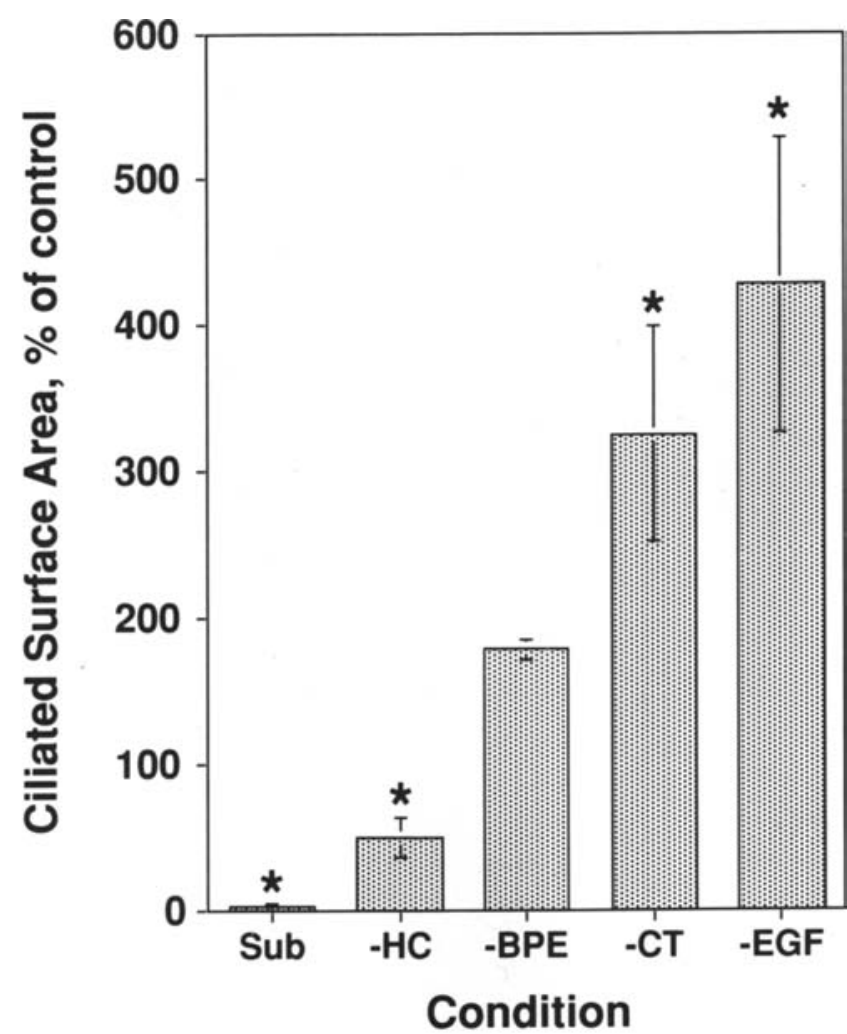

Figure 5. Effect of culture conditions on ciliated cell differentiation. RTE cells were cultured under different conditions and immunostained with mAb RTE3. The percentage of surface area covered by ciliated cells was estimated as described in MATERIALS AND METhods. Control cultures contained CM for the entire 14 days, and an air-liquid interface was established at day 7. Conditions shown are: Sub $=$ cells were grown submerged in CM for 14 days; $-\mathrm{HC}$ and $-\mathrm{CT}=$ cells were grown with an air-liquid interface in $\mathrm{CM}$ without $\mathrm{HC}$ or CT (days 0 through 14), respectively; -BPE and $-\mathrm{EGF}=$ cells were grown with an air-liquid interface in CM without BPE or EGF (days 7 through 14), respectively. Results from two experiments (one containing control, Sub, and - $\mathrm{HC}$ groups; one containing control, -BPE, -CT, and -EGF groups) are shown. Data are the average of 2 or 3 cultures per experiment, expressed as percentage of control cultures grown in parallel. Error bars indicate standard deviation (Sub, $-\mathrm{HC}$ ) or range (-BPE, $-\mathrm{CT},-\mathrm{EGF})$. * Significantly different from control at $P<0.05$ by Student's $t$ test.

visible cilia. Of the remaining $10 \%$ positive cells, almost all had abundant microvilli and probably represent preciliated cells. These results demonstrate that immunostaining with RTE3 is a reliable method to evaluate the extent of ciliated cell differentiation in RTE cultures.

\section{Mitogen Withdrawal Stimulates and Submersion Inhibits Ciliated Cell Differentiation}

To identify factors regulating ciliated cell differentiation, the effect of several modified culture conditions on ciliated cell development was examined. Control cultures of RTE cells were grown as described in CM with an air-liquid interface created on day 7 of culture. Parallel cultures were grown under the following conditions: (l) in CM but no air-liquid interface was created (i.e., submerged culture); (2) $\mathrm{HC}$ and (3) CT were omitted from the media at the start of the culture,

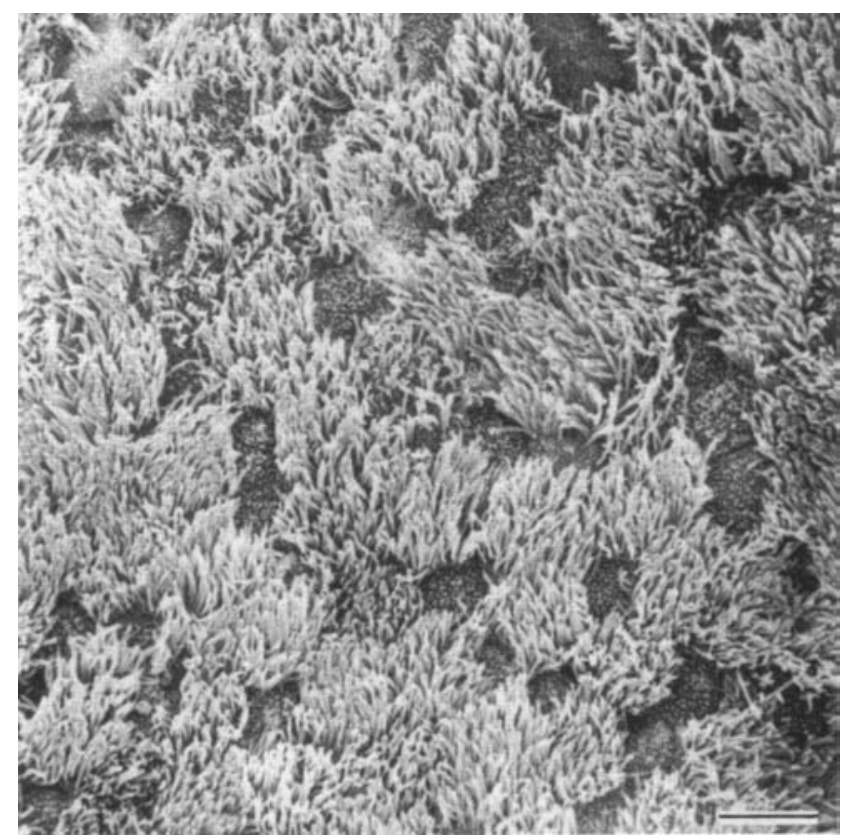

Figure 6. Scanning electron micrograph of a day 14 culture. RTE cells were grown in CM for 7 days and then for an additional 7 days at an air-liquid interface in CM lacking EGF. Abundant cilia are evident on the culture surface. Bar $=10 \mu \mathrm{m}$.

with an air-liquid interface created on day 7; (4) EGF and (5) BPE were removed from the media at day 7 and an airliquid interface was also created. Cultures were fixed at day 14 and immunostained with mAb RTE3 to determine the extent of ciliated cell differentiation. Cultures grown in the submerged state for the entire 14-day period contained very few stained cells compared with control cultures (Figures $4 a$ and $4 b)$. Cultures grown in media lacking the mitogenic compounds EGF, BPE, or CT were stained more heavily than those grown in CM (Figure 4c). The percentage of surface area stained with RTE3 was estimated using the image analysis program and normalized to control cultures from the same experiment. Figure 5 shows results obtained in two experiments, one that contained control, $-\mathrm{HC}$, and submerged cultures, and one that contained control, -EGF, -BPE, and -CT cultures. In these experiments, control cultures grown in $\mathrm{CM}$ averaged $3.5 \%$ and $8.6 \%$ ciliated surface area. Cultures that were submerged in $\mathrm{CM}$ throughout the culture period showed a 25 -fold decrease in ciliated cell differentiation and cultures grown in the absence of $\mathrm{HC}$ showed a 2-fold decrease, compared with parallel cultures grown in CM (Figure 5). In contrast, cultures grown in the absence of BPE, CT, or EGF demonstrated 2- to 4-fold increases in ciliated cell differentiation. Many areas of the cultures grown in the absence of EGF, CT, or BPE were heavily ciliated (Figure 6). In other experiments, RTE cells were grown in media lacking EGF, BPE, CT, or combinations of mitogenic factors. Although the actual percentage of surface area covered by ciliated cells varied between experiments, the largest increase in ciliated cells was observed when cells were cultured in media lacking both EGF and CT from the day the air-liquid interface was created (data not shown). Therefore, this condition was chosen to study the effect of mitogen withdrawal on ciliated cell differentiation in more detail. 
Figure 7. Effect of mitogen removal on ciliated cell differentiation, cell number, and DNA synthesis. RTE cells were grown for 7 days in $\mathrm{CM}$ and then for 7 additional days in $\mathrm{CM}$ with or without EGF and CT. A. At the days indicated, cultures were immunostained with RTE3 to determine the percentage of surface area covered by ciliated cells. B. In parallel cultures, cell number (squares) and DNA synthesis per $10^{4}$ cells (circles) was determined. Each point represents the average of three cultures \pm SD. Open symbols: control; shaded symbols: -EGF/-CT. * Significantly different from control at $P<0.05$ using the Student's $t$ test.
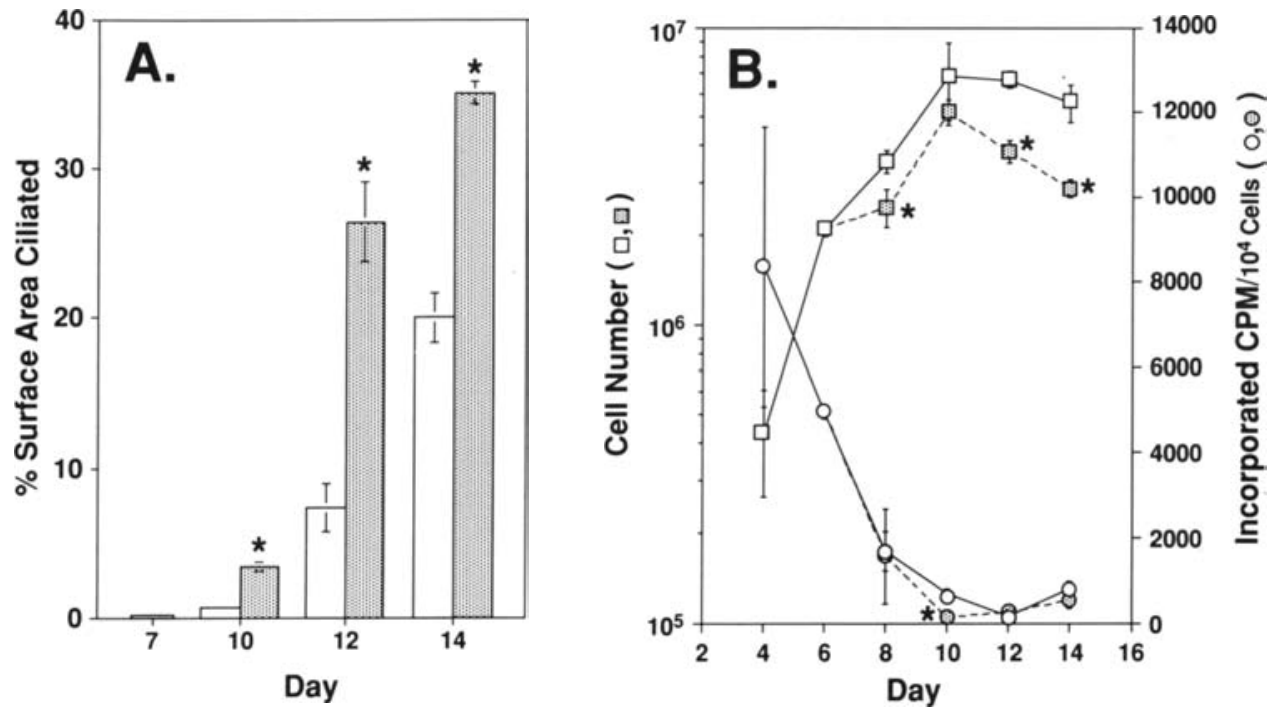

\section{Mitogen Removal Accelerates Ciliated Cell Development and Reduces Cell Proliferation}

Cultures of RTE cells were grown submerged in CM until the cells were confluent (day 7 or 8 of culture). An air-liquid interface was then created, and in one half of the cultures media was replenished daily with $\mathrm{CM}$, while in the other half of the cultures media was replenished with $\mathrm{CM}$ without EGF and CT (-EGF/-CT). Groups of three cultures from each condition were analyzed by RTE3 staining every 2 to 3 days until the end of the experiment. Cell number and DNA synthesis were measured in parallel cultures.

The removal of EGF and CT resulted in a more rapid increase in ciliated cell development; by 3 days, the $-\mathrm{EGF} /-\mathrm{CT}$ cultures showed a 5 -fold increase over controls in the percentage of surface area covered by ciliated cells (Figure 7A). After 5 and 7 days in the reduced growth factor media, the percentage of ciliated surface area was 4-fold and 2-fold greater, respectively, than in controls. About one third of the culture surface was covered by ciliated cells 7 days after removal of EGF and CT.

RTE cells cultured under control conditions undergo an initial period of rapid proliferation before reaching a plateau at between 2 to $8 \times 10^{6}$ cells per culture (Figure 7B). DNA synthesis was high early in culture and decreased by nearly two orders of magnitude as the cultures entered plateau phase. Removing EGF and CT from the media at day 7 resulted in a further decrease in DNA synthesis. At day 10, DNA synthesis in the -EGF/-CT cultures was 5-fold less than in the $\mathrm{CM}$ cultures $\left(1.31 \pm 0.5 \times 10^{2} / 10^{4}\right.$ cells versus $6.77 \pm 1.69 \times 10^{2} \mathrm{cpm} / 10^{4}$ cells). The decrease in DNA synthesis was followed by a decrease in cell number, so that by day 14 the $-E G F /-C T$ group of cultures contained about $50 \%$ fewer cells $\left(2.9 \pm 0.2 \times 10^{6}\right.$ cells in the $-\mathrm{EGF} /-\mathrm{CT}$ group versus $5.6 \pm 1 \times 10^{6}$ cells in the $\mathrm{CM}$ group). This experiment was repeated with the same results.

Because the removal of EGF and CT from the media caused a decrease in total cell number, the measurements of ciliated surface area may underestimate the effect of removing EGF and CT on ciliated cell differentiation. For example, if two cultures contained the same number of ciliated cells on the surface, but one contained twice as many cells, the percentage of ciliated cells could differ by $50 \%$. Therefore, the actual percentage of ciliated cells in the two groups was determined in an additional experiment. Cultures grown in $\mathrm{CM}$ and -EGF/-CT as above were dissociated enzymatically at day 14 , and cytospins were prepared. The cytospins were immunostained with RTE3, and the percentage of ciliated cells was determined. The -EGF/-CT cultures contained $33 \%$ ciliated cells, compared with only $8 \%$ in the CM cultures, indicating that withdrawal of mitogens probably increased ciliated cell differentiation to a greater extent than estimated by the immunostaining procedure.

To examine the effect of withdrawing EGF and CT from the media on the overall morphology of the cultures, thin sections were prepared from CM and -EGF/-CT cultures 7 days after creation of the air-liquid interface. Except for the increased number of ciliated cells in the -EGF/-CT culture, no obvious differences were observed between the two groups. Both cultures showed some variability in the thickness of the epithelium, with most areas of the culture membrane covered by a pseudostratified epithelium containing morphologically identifiable ciliated, secretory, and basal cells (Figure 8).

\section{Mitogen Removal Decreases Mucin Production}

It was observed during media changes that the $-E G F /-C T$ cultures appeared to be producing less mucus-like, viscous material on the apical surface than the CM cultures. Mucus production was therefore measured in an enzyme-linked immunosorbent assay using mAb RTE11, which has been shown to react with rat mucin $(18,19)$. The $-E G F /-C T$ cultures produced 2- to 3-fold less mucin than the control cultures $(0.7 \pm 0.1$ versus $2.3 \pm 0.2 \mu \mathrm{l}$ mucin equivalents at day 15; significantly different at $P<0.005$ ).

\section{Discussion}

The process of ciliogenesis in mammalian epithelium has been described in detail at the morphological level. For example, based on a series of electron micrographs taken during the development of fetal rat lungs, Sorokin (20) identified 

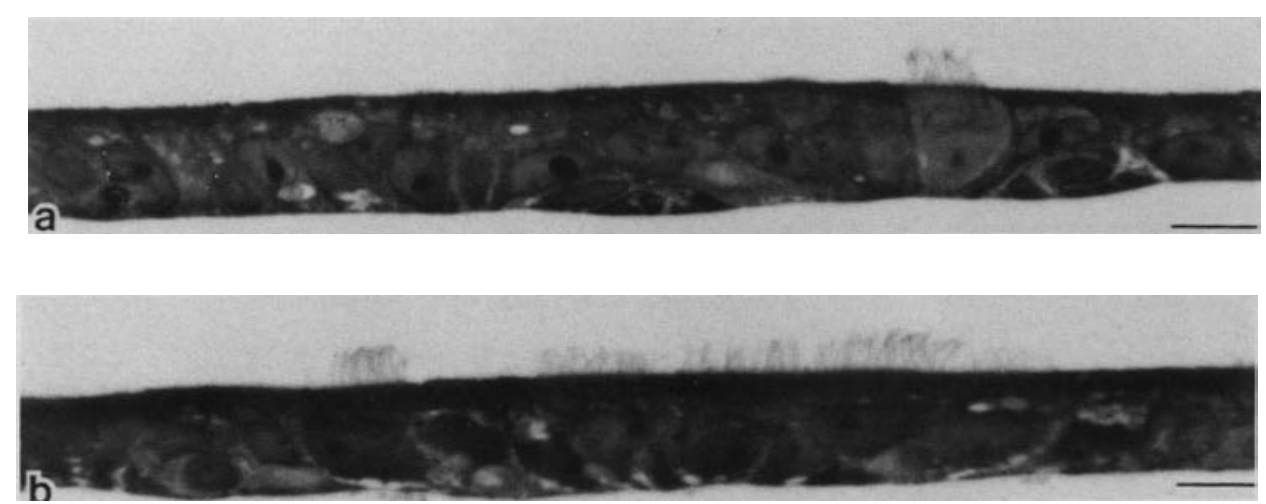

Figure 8. Morphology of 14-dayold air-liquid interface cultures. a. RTE cells were cultured in CM for 14 days. b. RTE cells were cultured in CM for 7 days and then in CM without EGF and CT from day 7 through 14 . The histology of the two cultures is similar except that more ciliated cells are evident in panel $b$. Lee's methylene blue-basic fuchsin stain, $1-\mu$ m-thick methacrylate sections. Bars $=10 \mu \mathrm{m}$. several stages in the formation of cilia. These included the appearance of fibrogranular aggregates, their maturation into deuterosomes, centrioles, and then basal bodies, and the assembly of the actual cilium (see also reference 21). However, the cellular pathway and the regulation of ciliated cell development in the airways is not well understood. This has been at least partially due to the lack of a suitable in vitro system that supports ciliated cell differentiation. Recently, a number of reports have described the presence of ciliated cells in cultured airway epithelium (9-15). Our laboratory has described a procedure in which RTE cells seeded on a collagen gel-coated membrane and cultured at an air-liquid interface differentiate into both secretory and ciliated cells (16). In this study, our goals were to develop a method to rapidly quantitate ciliated cell differentiation in RTE cultures and to identify major factors regulating ciliated cell development.

RTE3 was previously shown to react with the surface of ciliated cells in rat tracheobronchial epithelium (19). Ultrastructural immunocytochemistry demonstrated that RTE3 was highly specific for ciliated cells. RTE3 clearly labeled the apical plasma membrane, microvilli, and cilia of ciliated cells, with negligible labeling of nonciliated cells. Preciliated cells, identified by the presence of long, thin microvilli and basal bodies in the absence of mature cilia (13, $20,22,23$ ), were also lightly labeled by mAb RTE3; however, a more extensive study would be needed to conclude that the RTE3 antigen is expressed in preciliated cells. Other investigators have also described an antibody that recognizes ciliated cells in the absence of mature cilia (24). Immunostaining of RTE cultures with RTE3 and measuring the amount of stained surface area gave similar estimates of the extent of ciliated cell differentiation as more labor-intensive procedures such as SEM. The RTE3 immunostaining procedure allowed us to rapidly assess the effect of experimental variables on ciliated cell differentiation.

We observed that ciliated cell differentiation was inhibited by as much as 25 -fold when RTE cultures were submerged with approximately $0.1 \mathrm{ml}$ of media/ $\mathrm{cm}^{2}$. This agrees with a previous report by Kaartinen and colleagues (16) that ciliated cell differentiation in RTE cultures is inhibited by submersion. Other studies have also demonstrated increased ciliogenesis in air-exposed cultures $(11,12,14,15,25)$. However, ciliated cell differentiation has also been shown to occur in submerged cultures of hamster tracheal epithelial cells when grown on a collagen gel in the presence of conditioned media (26). Ciliated cells also develop in submerged cultures of human nasal epithelium when grown in suspension, but not when the same cells are cultured attached to a collagen gel (13). Ciliogenesis is also known to occur in fetal airways, which are submerged in fluid until birth (23). Thus, the effect of submersion on ciliated cell differentiation appears to be specific, both for the species and the particular culture conditions used. Additional studies will be necessary to understand the mechanism by which submersion inhibits ciliated cell differentiation.

Removal of $\mathrm{HC}$ from the media for the entire culture period resulted in an approximately 50\% decrease in the amount of ciliated surface area compared with control RTE cultures. Our observations are in agreement with the earlier report that $\mathrm{HC}$ also improved the morphological differentiation obtained in cultures of canine tracheal epithelium (9). However, the canine tracheal cells were plated at a high density $\left(1 \times 10^{6}\right.$ cells $\left./ \mathrm{cm}^{2}\right)$; therefore, the effect observed on canine cells may have been on maintenance of the differentiated phenotype and not on differentiation itself. It is interesting to note that steroid treatment has also been reported to increase ciliogenesis in asthma patients (27). Therefore, the possible role of steroids in ciliogenesis and ciliated cell differentiation should be examined further.

Removal of EGF, CT, or BPE from the culture media increased ciliated cell differentiation by 2 - to 4 -fold compared with cultures maintained in CM. Other investigators have used concentrations of growth factors similar to those in CM for culture of tracheal epithelial cells from several animal species, including dog, guinea pig, and human $(9,14,15)$. Some of these compounds have been examined for their overall effect on growth and differentiation of respiratory epithelium; however, these previous studies did not quantitate changes in the percentage of ciliated cells. In agreement with our results, media lacking EGF and CT was reported to be most effective at producing a pseudostratified epithelium in cultures of canine tracheal cells cultured on collagen matrices (9). Addition of CT alone was reported to have no effect on culture morphology; however, the addition of EGF produced a multilayering of squamous cells. In cultures of human tracheal epithelium, the use of a serum supplement or fetal calf serum improved growth and differentiation compared with that obtained with defined growth factors (15). 
These results indicate that high levels of growth-promoting factors may inhibit ciliated cell differentiation.

The removal of EGF and CT from the culture media resulted in a higher percentage of ciliated cells at each time point examined. DNA synthesis was significantly reduced in the $-\mathrm{EGF} / \mathrm{CT}$ groups at day 10. However, the observed increase in ciliated cell differentiation after withdrawal of mitogens may not be the direct result of a decrease in proliferation, because the rate of DNA synthesis decreases rapidly as confluence is attained in both the CM and the -EGF/ $-\mathrm{CT}$ cultures. The decrease in DNA synthesis was followed by a reduction of total cell number in the $-\mathrm{EGF} /-\mathrm{CT}$ groups. This decrease in cell number may be the result of increased ciliated (terminal) cell differentiation.

The mechanism(s) by which removal of growth factors leads to an increase in ciliated cell differentiation is unknown. However, in other systems, mitogenic compounds have been shown to have significant effects on differentiation. For example, depletion of mitogens from the media of myoblast cell lines results in muscle cell differentiation $(28,29)$. Terminal differentiation is inhibited by EGF in cultures of human (30) and mouse (31) keratinocytes. EGF is also known to have many other effects in addition to stimulating growth. For example, our laboratory has shown that EGF treatment of primary RTE cells increases fibronectin RNA and protein expression and induces organization of a fibrillar matrix (P. Ferriola and associates, unpublished data). In the present studies, the removal of EGF and CT decreased the secretion of mucus in addition to increasing the percentage of ciliated cells. Removing EGF from cultures of RTE cells has also been shown to reduce the number of secretory cells present in RTE cultures (K. Guzman and associates, unpublished data). An increase in ciliated cell differentiation while the number of secretory cells decreases is consistent with the hypothesis that secretory cells are the precursors for ciliated cells. Further studies will be needed to understand fully the mechanisms by which mitogen withdrawal regulates ciliated and/or secretory cell differentiation and the interrelationships between them.

The results presented here may lead to improvements in conditions for the culture of respiratory epithelium. Many of the factors commonly used in cultures of airway epithelial cells have been shown to increase proliferation. However, the effects of these compounds on differentiation in air-liquid cultures has not been carefully examined. The normal rat trachea has been reported to contain between $17 \%$ and $33 \%$ ciliated cells (32). In RTE cultures grown in the absence of EGF and CT, we observed $>30 \%$ ciliated cells, in good agreement with the in vivo estimates. These studies have also identified conditions that significantly reduce or enhance ciliated cell development, and will form the basis of further studies directed at understanding the regulation and pathway of ciliated cell differentiation.

In conclusion, these studies have identified submersion and mitogenic compounds as being key regulators of ciliated cell differentiation in vitro. Our results indicate that factors that stimulate proliferation of tracheobronchial epithelium may inhibit ciliated cell differentiation. Identification of other factors that regulate airway cell differentiation and their mechanism(s) of action may be useful in the treatment of some disease states.

Acknowledgments: The authors thank Dr. Pat Coin for assistance with the image analysis system, Vicky Madden for her expert assistance with the SEM work, and Dr. David Umbach for assistance with statistical analysis.

\section{References}

1. Lee, R. M. K. W., and J. B. Forrest. 1991. Structure and function of cilia. In The Lung: Scientific Foundations. R. G. Crystal and J, B. West, editors. Raven Press, New York. 169-181.

2. Diamond, G., D. E. Jones, and C. L. Bevins. 1993. Airway epithelial cells are the site of expression of a mammalian antimicrobial peptide gene. Proc. Natl. Acad. Sci, USA 90:4596-4600.

3. Rossi, G. A., O. Sacco, B. Balbi, S. Oddera, T. Mattioni, G. Corte, C. Ravazzoni, and L. Allegra. 1990. Human ciliated bronchial epithelial cells: expression of the HLA-DR antigens and of the HLA-DR alpha gene, modulation of the HLA-DR antigens by gamma-interferon and antigenpresenting function in the mixed leukocyte reaction. Am. J. Respir. Cell Mol. Biol. 3:431-439.

4. Sturgess, J. M. 1989. Ciliated cells of the lung. In Lung Cell Biology. D. Massaro, editor. Marcel Dekker, New York. 115-151.

5. Abdi, S., M. J. Evans, R. A. Cox, H. Lubbesmeyer, D. N. Herndon, and D. L. Traber. 1990. Inhalation injury to tracheal epithelium in an ovine model of cotton smoke exposure. Am. Rev. Respir. Dis. 142:1436-1439.

6. Carson, J. L., A. M. Collier, S. S. Hu, and R. B. Devlin. 1993. Effect of nitrogen dioxide on human nasal epithelium. Am. J. Respir. Cell Mol. Biol. 9:264-270.

7. Colizzo F., M. J. Krantz, J. E. Fish, and A. T. Hastie. 1992. Ciliated respiratory epithelial surface changes after formaldehyde exposure. J. Toxicol. Environ. Health 35:221-234.

8. Keenan, K. P., J. W. Combs, and E. M. McDowell. 1982. Regeneration of hamster tracheal epithelium after mechanical injury. Virchows Arch. B Cell Pathol. 41:193-214.

9. van Scott, M. R., N. P. Lee, J. R. Yankaskas, and R. C. Boucher. 1988. Effect of hormones on growth and function of cultured canine tracheal epithelial cells. Am. J. Physiol. 255(Cell Physiol. 24):C237-C245.

10. Baeza-Squiban, A., E. Boisvieux-ulrich, C. Guilianelli, O. Houcine, G. Geraud, C. Guennou, and F. Marano. 1994. Extracellular matrixdependent differentiation of rabbit tracheal epithelial cells in primary culture. In Vitro Cell. Dev. Biol. 30A:56-67.

11. Adler, K. B., P.-W. Cheng, and K. C. Kim. 1990. Characterization of guinea pig tracheal epithelial cells maintained in biphasic organotypic culture: cellular composition and biochemical analysis of released glycoconjugates. Am. J. Respir. Cell Mol. Biol. 2:145-154.

12. De Jong, P. M., M. A. J. A. Van Sterkenburg, S. C. Hesseling, J. A. Kempenaar, A. A. Mulder, A. M. Mommaas, J. H. Dijkman, and M. Ponec. 1994. Ciliogenesis in human bronchial epithelial cells cultured at the airliquid interface. Am. J. Respir. Cell Mol. Biol. 10:271-277.

13. Jorissen M., B. Van der Schueren, H. Van den Berghe, and J.-J. Cassiman. 1990. Ciliogenesis in cultured human nasal epithelium. ORL 52:368-374.

14. Whitcutt, M. J., K. B. Adler, and R. Wu. 1988. A biphasic chamber system for maintaining polarity of differentiation of cultured respiratory tract epithelial cells. In Vitro Cell. Dev. Biol. 24:420-428.

15. Yamaya, M., W. E. Finkbeiner, S. Y. Chun, and J. H. Widdicombe. 1992. Differentiated structure and function of cultures from human tracheal epithelium. Am. J. Physiol. 262(Lung Cell Mol. Physiol. 6):L713-L724.

16. Kaartinen, L., P. Nettesheim, K. B. Adler, and S. H. Randell. 1993. Rat tracheal epithelial cell differentiation in vitro. In Vitro Cell. Dev. Biol. 29A:481-492.

17. Barrow, R. E., C.-Z. Wang, R. A. Cox, and M. J. Evans. 1992. Cellular sequence of tracheal repair in sheep after smoke inhalation injury. Lung $170: 331-338$

18. Shimizu, T., P. Nettesheim, F. C. S. Ramaekers, and S. H. Randell. 1992. Expression of "cell-type-specific" markers during rat tracheal epithelial regeneration. Am. J. Respir. Cell Mol. Biol. 7:30-41.

19. Shimizu, T., P. Nettesheim, E. M. Eddy, and S. H. Randell. 1992. Monoclonal antibody (Mab) markers for subpopulations of rat tracheal epithelial (RTE) cells. Exp. Lung Res. 18:323-343.

20. Sorokin, S. P. 1968. Reconstruction of centriole formation and ciliogenesis in mammalian lungs. $J$. Cell Sci. 3:207-230.

21. Dirksen, E. R. 1991. Centriole and basal body formation during ciliogenesis revisited. Biol. Cell 72:31-38.

22. Moscoso, G., K. Nandra, and M. Driver. 1989. Ciliogenesis and ciliation of the respiratory epithelium in the human fetal cartilaginous trachea. Pathol. Res. Pract. 184:161-167.

23. Gaillard, D. A., A. V. Lallement, A. F. Petit, and E. S. Puchelle. 1989. 
In vivo ciliogenesis in human fetal tracheal epithelium. Am. J. Anat. 185:415-428.

24. Aitken, M. L., M. Villalon, M. Pier, P. Verdugo, and M. Nameroff. 1993. Characterization of a marker of differentiation for tracheal ciliated cells independent of ciliation. Am. J. Respir. Cell Mol. Biol. 9:26-32.

25. Kondo, M., W. E. Finkbeiner, and J. H. Widdicombe. 1991. A simple technique for culture of highly differentiated cells from dog tracheal epithelium. Am. J. Physiol. 261(Lung Cell. Mol. Physiol. 5):L106-L117.

26. Lee, T.-C., R. Wu, A. R. Brody, J. C. Barrett, and P. Nettesheim. 1984. Growth and differentiation of hamster tracheal epithelial cells in culture. Exp. Lung Res. 6:27-45.

27. Heino, M., J. Karjalainen, J. Ylikoski, A. Laitinen, and L. A. Laitinen. 1988. Bronchial ciliogenesis and oral steroid treatment in patients with asthma. Br. J. Dis. Chest 82:175-178.

28. Trouche, D., M. Grigoriev, J.-L. Lenormand, P. Robin, S. A. Leibovitch, P. Sassone-Corsi, and A. Harel-Bellan. 1993. Repression of c-fos pro- moter by MyoD on muscle cell differentiation. Nature 363:79-82.

29. Coppola, J. A., B. A. Lewis, and M. D. Cole. 1990 . Increased retinoblastoma gene expression is associated with late stages of differentiation in many different cell types. Oncogene 5:1731-1733.

30. Marchese, C., J. Rubin, D. Ron, A. Faggioni, M. R. Torrisi, A. Messina, L. Frati, and S. A. Aaronson. 1990. Human keratinocyte growth factor activity on proliferation and differentiation of human keratinocytes: differentiation response distinguishes KGF from EGF family. J. Cell. Physiol. 144:326-332.

31. Drozdoff, V., and W. J. Pledger. 1993. Commitment to differentiation and expression of early differentiation markers in murine keratinocytes in vitro are regulated independently of extracellular calcium concentrations. J. Cell Biol. 123:909-919.

32. Jeffery, P. K., and L. Reid. 1975 . New observations of rat airway epithelium: a quantitative and electron microscopic study. J. Anat. 120: 295-320. 